\title{
Dilemma: Where is the Old Happiness Road of "Aging Migration" -Based on Surveys in Some Areas of Xiamen
}

\author{
Lin $\mathrm{Hou}^{1,{ }^{*}}$ Liao anna ${ }^{1}$ Wang Jiaqi ${ }^{1} \mathrm{Hu}$ Zongchuan ${ }^{1}$ \\ ${ }^{I}$ School of Economics and Management, Xiamen University of Technology, Xiamen, Fujian 361024, China \\ *Corresponding autho. Email: 33811873@qq.com
}

\begin{abstract}
Compared with the "Beijing migrationers", a new special group appears--"Aging Migration"(Lao Piao). The problem of the lack of happiness of them is becoming increasingly obvious, and it has become a major problem that society cannot avoid. Taking some "Aging Migration"(Lao Piao) in Xiamen as the survey object, the research methods such as literature review and interview were used to investigate and classify them. We try to find the reasons for their lack of happiness and put up with related suggestions and measures for improving their happiness.
\end{abstract}

Keywords: Aging Migration, happiness, suggestions and measures

\section{INTRODUCTION}

As the process of urbanization continues to accelerate, migration of population has become increasingly apparent, and the pattern of rural-urban migration has become normal. The implementation of the two-child policy has prompted the elderly people to leave their hometown and follow their children to live in unfamiliar cities in order to take care of their grandchildren, which lead to a group"Aging Migration". "Aging" is the definition of age, "Migration" is the superficial characteristic of "separation of households", and "group" is a generalization of their homogeneity. Today, the elderly people are living longer; their health level are improving, and they have fewer children. Compared with the generation when children are clustered, they have more time and energy to care for their grandchildren. Due to traditional concepts and family responsibilities, they will leave rural areas for urban areas to care for their grandchildren. However, there are also passive situations. Their children have to turn to them for help because of insufficient living skills, high economic pressure, lack of child welfare and social trust. With the mutual influence of objective and subjective factors, the "Lao Piao" continued to grow. After retirement, they have to help their children to care for grandparents, which has almost become the most important job in their later years. In the process of accelerating social transformation and adapting to the new role of the "Lao Piao", the contradiction between the basic satisfaction of material conditions and lack of spirituality has become increasingly prominent. Although the elderly have a sense of enjoying around with their grandchildren, they are in dilemma and unhappiness because of lack of psychological comfort, transparent family identity, obvious urban-rural differences, barriers to inter-generational relationships, poor community friendship, difficult social integration, and lack of social welfare. The lack of happiness is significant and they do not know what to do.

This article takes some communities and parks in Xiamen as the research site, and takes the "Lao Piao" as the survey object. Based on the analysis of the literature and field visits, this article analyzes the phenomenon from the family, community, and government levels, and put forward some suggestions and measures to improve the happiness of the "Lao Piao".

\section{METHOD OF RESEARCH}

In order to comprehensively and truthfully reflect the current situation and feelings of "Lao Piao", We used the literature review method to make a preliminary understanding, and classified the "Lao Piao" by quantitative analysis method.We investigated the elderly who are far away from their hometown help with their children, and use the interview method to further explore the "Lao Piao" issue. A total of 100 people were interviewed, of which 80 were effectively interviewed.

\section{ANALYSIS OF DATA}

On the basis of recording and collating the contents of the interview and analyzing the literature, according to different natures, 80 effective interviews were classified into the "Lao Piao".

\subsection{Classified by “migration" bistance}

According to the distance from the migration of the elderly population, "Lao Piao" can be divided into "the provincial migration" group and "inter-provincial migration" group. The former is who migrate among different cities of a prov 
ince. The latter is who migrate from a province to another province. Through the interview, we found that most peopl e were from Fujian Province, among which Zhangzhou, L ongyan, and Sanming were the majority; while a few were from outside the province, including Henan, Shandong, Si chuan and so on. In the interviews, the "provincial migrati on "group had 61 people, accounting for $76.25 \%$, the "inter -provincial migration" had 19 people, accounting for 23.7 $5 \%$.

\subsection{Classified by "migration" time}

According to the length of the elderly population's migration time, the " Lao Piao" can be divided into "longterm migration" and "short-term migration". The shortterm and long-term time limit is separated by two years. The elderly who come to the urban areas within two years (including the year) are called "short-term migration"; the elderly who come to the city for more than two years are called "long-term migration". We found that thanks to the implementation of China 's two-child policy, there are many family choose to born the second child, which lead to the elderly people have to migrate again. This has become the most important factor affecting the long-term migration. In the interviews, there were 52 people belonging to the former, accounting for $65 \%$, and 27 people belonging to the latter, accounting for $35 \%$.

\subsection{Classified by "migration" subject}

According to the number of mobile subjects in the elderly population, the "Lao Piao" can be divided into single migration and double migration. The one who come to the children's city alone to take care of the grandchildren is called "single migration"; the two elderly who come to the children's city together are called "double migration". In the interview, there were 70 "double migration", accounting for $87.5 \%$, while only 10 "single migration", accounting for $12.5 \%$.

\subsection{Classified by "migration" will}

Based on the difference of the elderly population's will to look after grandchildren, the "Lao Piao " can be divided into " responsibility migration" and "family assistance migration". Then setting up the interview question of "the old people's views on taking care of their grandchildren", According to their answers, the elderly who hold the position of taking grandchildren as a duty and responsibility are called "responsibility migration" .Elderly people who think they are simply out of affection and come to care for their grandchildren are called "family assistance migration".In the interview, the number of "responsibility migration" is as high as 75 , accounting for $93.75 \%$. The "family assistance migration" are only 5 people, accounting for $6.25 \%$.

\section{CAUSES OF MISSING HAPPINESS}

\subsection{Personal causes}

\subsubsection{Rural sentiment is deeply solidified, anxious to return home as soon as possible}

Chinese traditional culture emphasizes the homeland complex of Antu's relocation. Especially for the elderly, it is the happiest thing to be able to spend their old age with the people they know best. However, social and economic development has promoted urbanization and population migration, and most young people have settled in different places. The various pressures in reality have caused the "Lao Piao" to be forced into the city, seemingly simple spatial displacement, but they are experiencing psychologically insurmountable barrier, living in the city, but the heart is in the hometown.

\subsubsection{Non-owner and non-object transparency, no time for children}

\begin{abstract}
"You stand firm in the big city, but they have to migrate." "Lao Piao" used to be the core of the family and had absolute speaking right. Now, in order to take care of their grandchildren, living with their children, they are neither the owner nor the guest. In addition to looking after the grandchildren and doing housework, it's hard to get involved other things, just like the "invisible person" in the family. It is inevitable that there is a lack of identification with the home. The implementation of the two-child policy today means that most of the "Lao Piao" have to "work" again with contradiction. Perhaps companionship is the most effective stabilizer, but children are busy with work and most of their free time is spent on their children. Too little concern for parents, fail to talk to them, understand their parents' thoughts, timely resolve the parents' worries and doubts, ignored the lack of parents' inner emotions, and fail to encourage them to go out and make friends, resulting in the psychological empty nest of the "Lao Piao", that seriously neglect the role of the family in helping the "Lao Piao" to adapt to urban life.
\end{abstract}

\subsubsection{Different ideology, difficult to bridge the gap between generations}

Intergenerational relations are always a big problem for society and families. In the era when the "Lao Piao" was young, as the focus of the family, they supported the family and raised their children. But over time, the children form their own families. The "Lao Piao" take care of their grandchildren, but they seem to be an "adjunct" to their children's family. Moreover, with the development of society, the new era belongs to the era of young people, 
and the "Lao Piao" is already a group that will soon be eliminated by the times, and it has gradually become a marginal figure in society and families. Therefore, the "Lao Piao" who help to take care of their grandchildren and are out of touch with the society and live together with their children often have problems in the intergenerational relationship due to their ideology, values and lifestyles.

\subsection{Social causes}

\subsubsection{Rapid urban process, difficult for rural parents to adapt}

The bustling and lively city is pretty different from the simple life scene in the countryside. From the familiar living environment to the unfamiliar city life, the "Lao Piao" will inevitably be affected by regional differences and silently give themselves a psychological hint as a stranger. The issue of adapting to the environment is the primary problem faced by the "Lao Piao" in urban life. Among them, which make "Lao Piao" helpless, The first is urban traffic. It is common for roads and residential areas to become parking lots. The second is the climate. The temperature difference between north and south is great in winter."Lao Piao" in the north who enjoys central heating, what the south felt is the cold and bitter wind, it is naturally difficult to adapt. The cities have high-rise buildings, heavy traffic, and poor air quality and even noisy until late at night, while the rural air is fresh and all is still when the night falls. Such a huge difference can easily lead to the "Lao Piao" with poor adaptability.

\subsubsection{Superficial interpersonal relationships make it difficult to integrate urban life}

The community is the place where urban residents live and do some activities. In this small social area, the "Lao Piao" belongs to external population. It should have benign interaction with the community environment and have a better living condition. However, It has difference between the community residents and "Lao Piao" because of their character, language, customs, cultural level and ideology, which leads to some inferiority complex. They are unwilling to take the initiative to communicate with others and with low enthusiasm, when contacting with others they will appear a sense of powerlessness, and the communication objects become closed and involute. The main task of the "Lao Piao" is to take care of their children and grandchildren in the city. They have few personal hobbies, and are easily occupied by housework even if they have free time, so less opportunities to participate in community activities. Furthermore, the social activities between urban and rural areas are great different, and there are many entertainment activities in urban elderly communities, such as square dancing, singing, and playing Tai Chi. The "Lao Piao" may have no talent or strong intention to perform, so the participation in community activities is low, and repeating the dull life.

\subsubsection{The welfare mechanism needs to be improved, and basic rights and interests cannot be guaranteed}

Most of the social welfare system in China is linked to the household registration system. The "Lao Piao" move to cities with their children, and the old-age welfare guarantees for medical care are still in the countryside. The welfare benefits in the city are still blank, and they cannot enjoy the elderly in the city. Preferential policies for people. Elderly residents of urban hukou enjoy the benefits of free bus rides, free or reduction of entrance fees to attractions, but they are not able to cover "Lao Piao". The off-site welfare guarantee system is still limited to the hukou registration framework. Medical expenses are expensive, reimbursement is small and difficult, and most matters need to be returned to the hometown. The procedures are complicated and cumbersome, which has brought various inconveniences to "Lao Piao" for medical treatment in different places.

\section{SUGGESTIONS AND COUNTERMEASURES TO IMPROVE "HAPPINESS"}

Although the problems of the "Lao Piao" are endless, its scale is still expanding. It is imperative to take measures to solve the problem of "Lao Piao". Propose solutions from the individual, family, community, and government to relieve the conflict So that they can get a solid "happiness".

\subsection{Actively seeking value to enrich one's life}

For the "Lao Piao" individuals, they should actively play the role of residual light, actively participate in social activities to exert their own value, and participate in community volunteer activities such as garbage sorting and transportation guidance in their free time; "live to learn old" Attend the university interest training class for the elderly. At the same time, the elderly should change their mindset, actively adapt to the surrounding unfamiliar environment, and find ways to resolve their emotions. For example, mingle with the square dance aunt, play chess with the uncles, meet each other, and meet the players The activity center is happy, participates in community entertainment activities, expands the social circle, and enriches their lives; make friends, and share their experience of taking the baby with "Bao Ye" and "Bao Milk", and talk to each other and solve common problems. 
can truly feel the sense of belonging at home and obtain the actual happiness, also requires the cooperation of the family, the community and the government.

\subsection{Children's diligence and thoughtful consolation}

The emotional communication between children and parents in the family has a great shaping and penetrating power on the old man's psychology. The "Lao Piao" left their hometown and came to a strange city. They had a lot of maladjustments and needed more emotional care and The value is affirmed. Create a family fusion culture, "culture back", teach them to use emerging products to solve practical problems, and help them adapt to the city's environment and lifestyle faster; in the leisure time, communicate with the elderly and listen to their hearts. Needs, give them enough space to develop personal interests, make friends, and avoid the idea of "nanny" parents.

\subsection{The community actively builds a platform to create a good atmosphere}

The community is a family-based social community and a space for living and entertaining the residents of the community. As an important part of the new members of the city, it is difficult to integrate into the community due to various restrictions. Rebuild the organization and improve the community activity platform, hold more community activities to invite "Lao Piao" to participate and improve the sense of belonging to the community; restructure the community's grass-roots social network, regularly conduct counseling services, provide material and spiritual "dual" condolences, and create a good and harmonious care for "Lao Piao" community atmosphere.

\subsection{The government needs to improve the system and provide tangible benefits}

The "Lao Piao" has separated their residence from their household registration due to living in different places with their children. As a result, they often do not enjoy the benefits of the government. Therefore, the government should adjust the old-age and medical insurance welfare protection system for this group and introduce corresponding settlements. Policies, relax the threshold of household registration, reduce regional differences, promote the reform of the household registration system, and promote the dual linking of household registration and welfare. At the same time, guide the mass media to pay more attention to special groups such as "Lao Piao" and get enough attention from society to establish "Lao Piao" Re-socialized social environment.

\section{CONCLUSION}

How to get the "Aging Migration"(Lao Piao) out of the dilemma, adapt to and integrate into the new city, the new community and the family of their children, so that they

\section{ACKNOWLEDGMENT} Technology's innovation training program for undergraduate in 2019.

\section{REFERENCES}

[1] Guo Zhiqian. "migrationing": The Mobile Practice of the "Lao Piao" in the Countryside--A Case Study Based on Shanxi Yao Village [J].Journal of Shanxi Agricultural University (Social Science Edition), 2016,15 (12): 864-868.

[2] Wang Xinyu, Li Xiaochun. Retirement of Old People in the Old People in the Process of Urbanization [J].Population and Society, 2017,33 (4): 50-58.

[3] Tang Yuanjun, Sun Yanli, Li Zhenjie, et al. Research and countermeasure analysis on social integration of "Lao Piao" in cities in southern Jiangsu [J]. Science, Education and Culture (Late Issue), 2017 (12): 191-192.

[4] Cao Guojiao, Nie Qingyan. Research on Social Adaptation of "Lao Piao" from the Perspective of Social Integration [J]. Future and Development, 2017,41 (11): 66-69.

[5] Song Jiahang, Ye Wenjie, Xie Jing. Adaptation, Integration and Energization: Three Key Issues of "Lao Piao" to Improve the Quality of Life [J].Weishi (Modern Management), 2017 (09): 61-62.

[6] Zhang Liwen, Gou Yanfeng. A Study on the Physical Practice of the "Lao Piao" in Xi'an from the Perspective of Sociological Sociology [J]. Legal System and Society, 2018 (17): 171-172.

[7] Yang Li, Xu Qingqing. Dynamics of Social Adaptation and Duality of Emotional Experience-Based on the Qualitative Study of Lao Piao in Shanghai [J]. Aging Science Research, 2018,6 (06): 45-58.

[8] Fang Jingrui. Structural contradictions and coping paths formed by "Lao Piao"[J]. Journal of Bengbu University, 2018, 7 (01): 94-97.

[9] Yang Juhua. Migrant Generations in Migrant Times: Analysis of Multidimensional Features of Older Migrant Populations [J]. Journal of Demography, 2018, 40 (04): 43-58.
This paper was the outcome of Xiamen University of 